\title{
Drug-induced Angle-Closure Glaucoma
}

\author{
Aruj K Khurana, Bhawna Khurana, Ashok K Khurana \\ Regional Institute of Ophthalmology, Post Graduate Institute of Medical Sciences, Rohtak, Haryana, India
}

\begin{abstract}
Correspondence: Ashok K Khurana, Senior Professor, Regional Institute of Ophthalmology, Post Graduate Institute of Medical Sciences, Rohtak, Haryana, India
\end{abstract}

\section{ABSTRACT}

Drug-induced angle-closure glaucoma is an important entity for the ophthalmologist as well as the general physician as it represents a preventable cause of potential blindness. This brief review highlights the fact that a high index of suspicion, in a susceptible individual followed by confirmation on appropriate imaging modality (UBM, ultrasound or anterior segment OCT) can alleviate the threat to sight and also help to institute appropriate therapy.

Keywords: Acute angle closure, Drug-induced glaucoma.

\section{INTRODUCTION}

Drug-induced angle-closure glaucoma is an important entity for the ophthalmologist as well as the general physician as it represents a preventable cause of potential blindness. ${ }^{1}$ Acute angle-closure glaucoma can develop in a susceptible individual by various classes of drugs. ${ }^{2}$ Practitioners using any of these drugs should be aware of their potential to cause acute angle closure, such that a patient presenting with signs or symptoms of acute angle closure should be immediately referred to an ophthalmologist. ${ }^{3}$ Drugs implicated in the causation of acute angle-closure glaucoma (AACG) include sulfa-based drugs, adrenergic drugs and drugs with anticholinergic effects. It is important to understand that the mechanism of causation of AACG is different for each of these drugs because the management strategy differs as per the pathophysiology. Ultrasound biomicroscopy helps in clinching the diagnosis by ruling out other causes of shallow anterior chamber, such as accommodative spasm and primary angle closure. ${ }^{4}$

\section{Mechanism of Drug-induced AACG}

Sulfa drugs like topiramate, a sulfamate substituted monosaccharide antiepileptic agent precipitates AACG by way of inducing ciliary body edema. The underlying mechanism has been well characterized by ultrsound technology. Drug-induced changes in membrane potential have been hypothesized to cause ciliary body edema, ${ }^{7}$ leading to relaxation of zonules and resultant lens thickening. Anterolateral rotation of the ciliary body about its attachment to the scleral spur leads to anterior displacement of the lens and iris and concomitant shallowing of anterior chamber. Concomitant choroidal detachment and supraciliary effusion are known to occur. The fact that effusion occurs only in a few patients taking topiramate and, more importantly, it typically occurs on doses well within the normal therapeutic range and in patients with normal anterior chamber depth suggests an idiosyncratic etiology. ${ }^{8}$ No known risk factors exist for this syndrome. ${ }^{9}$ Other sulfa-based drugs known to be associated with AACG: Acetazolamide, hydrochlorothiazide and cotrimoxazole. ${ }^{10}$

Anticholinergic drugs implicated in the causation of AACG include atropine, homatropine, cyclopentolate and ipratropium bromide. ${ }^{10,11}$ Atropine is often used to treat bradycardia, especially related to general anesthesia. Postoperative AACG has been reported in patients after general anesthesia for abdominal, orthopedic, facial and endoscopic surgery. ${ }^{12}$

Botulinum toxin has been reported as a cause of AACG. The mechanism was postulated as diffusion toward the ciliary ganglion and impedance of cholinergic innervation of the pupil, following its injection around eyelid for blepharospasm. ${ }^{13}$

Cholinergic agents, such as pilocarpine, acetylcholine and carbachol, can induce AACG by causing forward movement of iris lens diaphragm, ${ }^{10}$ especially in eyes with zonular weakness and exfoliation syndrome. ${ }^{14}$

Adrenergic agents can precipitate an attack of AACG through ocular $^{15}$ as well as systemic route of administration (surgical anesthesia, anaphylactic shock, ventricular fibrillation, nasal administration for epistaxis and bronchodilators). Ocular agents cause mydriasis which can precipitate an attack of acute AACG in predisposed individuals with shallow anterior chambers. ${ }^{5}$ Stimulation of ciliary body beta- 2 receptors increases aqueous secretion. Some drugs have an indirect sympathomimetic activity that can induce AACG, such as amphetamines, antidepressants and cocaine. ${ }^{6}$

Antidepressants, such as tri and tetracyclic depressants and SSRIs, have been associated with AACG in susceptible individuals due to their cholinergic action. ${ }^{16}$ Supraciliary effusion seen on ultrasonography has been identified as the pathogenetic mechanism. ${ }^{17}$

Anticoagulant therapy, in the form of heparin as well as low molecular weight heparin (enoxaparin, warfarin), can cause 
AACG by inducing massive vitreous, choroidal or subretinal hemorrhage. Risk factors for the same include anticoagulants, exudative age related macular degeneration and nanophthalmos. $^{18}$

Histamine $\mathrm{H} 1$ and $\mathrm{H} 2$ receptor antagonists, such as chlorpheniramine and cimetidine respectively, have a weak anticholinergic effect, which can induce mydriasis and AACG in predisposed patients. ${ }^{19}$

Investigative modalities for diagnosis of drug-induced AACG include high frequency ultrasound biomicroscopy, anterior segment ocular coherence tomography and B-scan ultrasound. They help to establish and document the underlying mechanism $^{20}$ and prognostication of drug-induced AACG. Patients with severe ciliochoroidal effusion on ultrasound $B$ scan have been noted to have the severe visual loss, highest myopic reading and maximum increase in intraocular pressure. ${ }^{21}$ Fluorescein angiography further helps to confirm the diagnosis by demonstrating transient lobular choriocapillaris hypoperfusion related to choroidal thickening. ${ }^{22}$

Management of drug-induced AACG depends on the underlying pathogenic mechanism and the presence or absence of pupillary block, which helps us to decide whether peripheral iridectomy would be curative or not.

Management of topiramate related AACG requires stopping the drug in concert with the prescribing physician, because decreasing the dose by as little as $50 \mathrm{mg}$ may exacerbate preexisting systemic conditions. ${ }^{23}$ In all reported cases, none has subsided without discontinuation of the drug. It is important to realize that most glaucoma cases resolve without miotics or iridotomy because pupillary block is not the cause of angle closure. ${ }^{21}$ Topical cycloplegic agents probably lower intraocular pressure by retracting the ciliary processes, along with topical-blockers and oral pressure-lowering agents. If unrecognized as a drug-related event, serious outcomes can occur (seven cases of permanent visual loss have been reported). ${ }^{3}$ For severe cases associated with very high intraocular pressures, the combination of mannitol and methylprednisolone can induce a rapid improvement which suggests the inflammation may be a part of the pathogenesis of topiramate induced AACG. ${ }^{24}$

Similarly in case of anticoagulants, the drug needs to be discontinued along with AACG management. Surgery may be required to drain choroidal effusion or hemorrhage. ${ }^{25}$

Prevention of drug-induced AACG, unfortunately does not have a straight forward solution because patients who are prescribed these drugs by physicians are not routinely sent to an ophthalmologist for a prior complete eye examination including gonioscopy to rule out preexisting narrow angles. Referring each patient for a prior ophthalmic evaluation may not be practically feasible. However, with increasing recognition of this entity, it is important for the physician to be on the look out for a predisposed patient by way of clues, such as patients wearing thick glasses that magnify objects suggesting a hypermetropic error. Ophthalmological consultation is warranted in a predisposed patient before starting treatment with drugs capable of potentiating AACG. ${ }^{26}$ A quick lateral torch light examination can also be carried out to rule out narrow angles. Ates et al recommend practising an oblique penlight illumination test by anesthesiologists to estimate anterior chamber depth and determine the population at risk before administering anticholinergic anesthetic drugs. ${ }^{27}$ Patients at risk for AACG in the postoperative period can be administered topical pilocarpine therapy to prevent any attack. Since symptoms of AACG may be overlooked or misinterpreted in a sedated or comatose patient, any patient who has a red eye and a subjective vision loss postoperatively should be examined urgently. ${ }^{3}$

Most importantly, if a patient on any of the implicated drugs reports back to the physician with symptoms suggestive of AACG, such as acute painful red eye or blurred vision, an immediate ophthalmological referral is warranted.

\section{CONCLUSION}

Drug-induced acute angle-closure glaucoma is a potentially avoidable cause of vision loss which requires vigilance on part of the physician and the ophthalmologist. A high index of suspicion, in a susceptible individual followed by confirmation on appropriate imaging modality (UBM, ultrasound or anterior segment OCT) can alleviate the threat to sight and also help to institute appropriate therapy.

\section{REFERENCES}

1. Razeghinejad MR, Myers JS, Katz LJ. Iatrogenic glaucoma secondary to medications. Am J Med Jan 2011;124(1):20-25.

2. Berdy JG, Berdy SS, Odin LS, et al. Angle closure glaucoma precipitated by aerosolized atropine. Arch Intern Med 1992;151;1658-60.

3. Lachkar Y, Bouassida W. Drug-induced angle-closure glaucoma. Curr Opin Ophthalmol 18:129-33.

4. Natesh S, Rajashekhara SK, Rao ASD, Shetty B. Topiramateinduced angle closure with acute myopia, macular striae. Oman J Ophthalmol Jan-Apr 2010;3(1):26-28.

5. Lachkar Y, Migdal C, Dhanjil S. Effect of brimonidine tartrate on ocular hemodynamic measurements. Arch Ophthalmol 1998;116:1591-94.

6. Wilcsek GA, Vose MJ, Francis IC, et al. Acute angle-closure glaucoma following the use of intranasal cocaine during dacryocystorhinostomy. Br J Ophthalmol 2002;86:1312.

7. Levy J, Yagev R, Petrova A, et al. Topiramate-induced bilateral angle-closure glaucoma. Can J Ophthalmol 2006;41:221-25.

8. Asconape JJ. Some common issues in the use of antiepileptic drugs. Semin Neurol 2002;22:27-39.

9. Rhee DJ, Ramos-Esteban JC, Nipper KS. Rapid resolution of topiramate induced angle-closure glaucoma with methylprednisolone and mannitol. Am J Ophthalmol 2006;141: 1133-34.

10. Tripathi RC, Triathi BJ, Haggerty C. Drug-induced glaucomas: Mechanism and management. Drug Saf 2003;26:749-67.

11. De Saint Jean M, Boursier T, Borderie V. Acute angle-closure glaucoma after treatment with ipratropium bromide and salbutamol aerosols. J Fr Ophthalmol 2000;23:603-05. 
12. Lentschener C, Ghimouz A, Bonnichon P, et al. Acute postoperative glaucoma after nonocular surgery remains a diagnostic challenge. Anesth Analg 2002; 94:1034-35.

13. Corridan P, Nightingale S, Mashoudi N, et al. Acute angle-closure glaucoma following botulinum toxin injection for blepharospasm. Br J Ophthalmol 1990;74:309-10.

14. Ritch R, Lowe RF. Angle-closure glaucoma: Clinical types. In: Ritch R, Shields MB, Krupin T (Eds). The glaucomas. St Louis: Mosby 1996;829-30.

15. Wolfs RC, Grobbee DE, Hofman A, et al. Risk of acute angleclosure glaucoma after diagnostic mydriasis in nonselected subjects: The Rotterdam study. Invest Ophthalmol Vis Sci 1997;38:2683-87.

16. Epstein NE, Goldbloom DS. Oral imipramine and acute angleclosure glaucoma. Arch Ophthalmol 1995;113:698.

17. Zelefsky JR, Fine HF, Rubinstein VJ. Escitalopram-induced uveal effusions and bilateral angle closure glaucoma. Am J Ophthalmol 2006;141:1144-47.

18. Schlote T, Freudenthaler N, Gelisken F. Anticoagulative therapy in patients with exudative age-related macular degeneration: Acute angle-closure glaucoma after massive intraocular hemorrhage. Ophthalmology 2005;102:1090-96.

19. Fraunfelder FW, Fraunfelder FT. Adverse ocular drug reactions recently identified by the National Registry of drug-induced ocular side effects. Ophthalmology 2004;111:1275-79.
20. Chalam KV, Tillis T, Syed F, Agarwal S, Brar VS. Acute bilateral simultaneous angle closure glaucoma after topiramate administration: A case report. Journal of Medical Case Reports 2008;2:1.

21. Singla Ritesh, Goyal Vippan, Singla Gaurav, Gupta Pooja, Kumar Rajiv. Acute ocular side effects of topiramate. Journal of Clinical and Diagnostic Research October 2011;5(5): 1082-85.

22. Pierre Blain, Michel Paques, Pascale Massin, Ali Erginay, PierreYves Santiago, Alain Gaudric. Acute transient myopia induced by indapamide. Am J Ophthalmol 2000;129:538-40.

23. Fraunfelder FW, Fraunfelder FT, Keates Edwin U. Topiramateassociated acute, bilateral, secondary angle-closure glaucoma. Ophthalmology 2004;111:109-11.

24. Rhee Douglas J, Ramos-Esteban Jerome C, Nipper Karen S. Rapid resolution of topiramate-induced angle-closure glaucoma with methylprednisolone and mannitol. Am J Ophthalmol 2006;141:1133-34.

25. Banta JT, Hoffman K, Budenz DL, et al. Presumed topiramateinduced bilateral acute angle-closure glaucoma. Am J Ophthalmol 2001;132:112-14.

26. Costagliola C, Parmeggiani F, Sebastiani A. SSRIs and intraocular pressure modifications: Evidence, therapeutic implications and possible mechanisms. CNS Drugs 2004;18:475-84.

27. Ates H, Kayikc,ioglu O, Andac, K. Bilateral angle-closure glaucoma following general anesthesia. Int Ophthalmol 2001;23:129-30. 